EPJ Web of Conferences 60, 15006 (2013)

DOI: $10.1051 /$ epjconf $/ 20136015006$

(C) Owned by the authors, published by EDP Sciences, 2013

\title{
Heavy-flavour measurements in proton-proton collisions with ALICE at the LHC
}

\author{
Markus Fasel ${ }^{1, a}$ on behalf of the ALICE Collaboration \\ ${ }^{1}$ Physikalisches Institut, Ruprecht-Karls Universität Heidelberg, Im Neuenheimer Feld 226, 69120 Heidelberg
}

\begin{abstract}
The measurement of heavy-flavour production in proton-proton collisions serves as a precise test for perturbative QCD. Furthermore it provides the reference for the corresponding studies in heavy-ion collisions. The measurements are performed by ALICE in hadronic channels as well as in the semi-electronic decay channel at midrapidity, profiting from the excellent particle identification capabilities and the good impact parameter resolution, and in the semi-muonic channel at forward rapidity. In particular, electrons from beauty hadron decays can be measured at low $p_{\mathrm{T}}$ by selecting electrons with a large distance to the primary vertex or by using electron-hadron correlations. Furthermore ALICE provides the unique opportunity to study the D meson yields as function of the multiplicity. We present heavy- flavour measurements in the various channels in pp collisions at $\sqrt{s}=7 \mathrm{TeV}$ and $\sqrt{s}=2.76 \mathrm{TeV}$, and discuss the results by comparing them to perturbative QCD calculations.
\end{abstract}

\section{Introduction}

The measurement of the production of heavy-flavour hadrons in proton-proton (pp) collisions serves as a precise test for perturbative QCD. Furthermore it provides the reference for studies in heavy-ion collisions, where a deconfined medium, the Quark-Gluon Plasma, is supposed to be formed. In addition, the investigation of heavy-flavour production as function of the charged particle multiplicity of the event may provide insights into multiple hard parton scatterings.

The measurements are performed with ALICE at the LHC. The experiment is described elsewhere in detail [1]. In the central barrel $(|\eta|<0.8)$, tracks are reconstructed using the Time Projection Chamber (TPC) and the Inner Tracking System (ITS), which also plays a major role in the primary vertex reconstruction and the reconstruction of secondary vertices. In addition, the ITS helps improving the track impact parameter resolution. Particle identification information are obtained using the TPC, the Time-OfFlight Detector (TOF), and in the electron measurement in addition from the Transition Radiation Detector (TRD) and the Electromagnetic Calorimeter (EMCal). The detectors are located in a homogeneous magnetic field of $0.5 \mathrm{~T}$. In forward direction $(2.5<\mathrm{y}<4.5)$, the muon spectrometer provides muon tracking and triggering.

Heavy-flavour production can be studied either in hadronic channels, where the charmed mesons are directly reconstructed via their invariant mass, or inclusively in the semi-leptonic channels, where the lepton samples contain background from different sources which has to be estimated and subtracted. With ALICE, heavy-flavour pro-

\footnotetext{
a e-mail: mfasel@physi.uni-heidelberg.de
}

duction is measured at midrapidity in the hadronic channels listed in Sec. 2 and in the semi-electronic channel discussed in Sec. 3, and at forward rapidity in the semimuonic channel (Sec. 3).

A good impact parameter resolution is crucial for the heavy-flavour analysis in order to separate the products from heavy-flavour hadron decays from the primary vertex and to separate the contributions from charm and beauty hadron decays, since the $c \tau$ of charm hadrons is $100-300$ $\mu \mathrm{m}$ and the $\mathrm{c} \tau$ of beauty hadrons is of the order of $500 \mu \mathrm{m}$. With the impact parameter resolution achieved (i.e. $75 \mu \mathrm{m}$ at $\left.p_{\mathrm{T}}=1 \mathrm{GeV} / c\right)$, ALICE is well-suited for heavy-flavour measurements.

\section{Measurement in the hadronic channels}

The differential cross section has been measured for $D^{0}$, $D^{+}, D^{*+}$ and $D_{s}^{+}$mesons in pp collisions at $\sqrt{s}=7 \mathrm{TeV}$ in the channels $D^{0} \rightarrow K^{-} \pi^{+}, D^{+} \rightarrow K^{-} \pi^{+} \pi^{+}, D^{*+} \rightarrow$ $K^{-} \pi^{+} \pi^{+}$, and $D_{s}^{+} \rightarrow \Phi \pi^{+} \rightarrow K^{+} K^{-} \pi^{+}$(and charge conjugates) $[6,7]$. The resulting cross sections are compared to fixed order plus next-to-leading logarithms (FONLL) [2-4] and general-mass-variable-flavour-number-scheme (GM-VFNS) [5] pQCD predictions. As example, the comparison of the differential production cross section of $D^{0}$ mesons with different calculations is shown in Fig 1 (left). The data is well described by pQCD calculations, typically at the upper limit of the theoretical uncertainty band when comparing to FONLL and at the lower limit when comparing to GM-VFNS. As it was reported recently, the data are also in good agreement with the $k_{T}$-factorization approach [8]. By extrapolating the measured cross section to the full phase space using FONLL pQCD calculations, the 

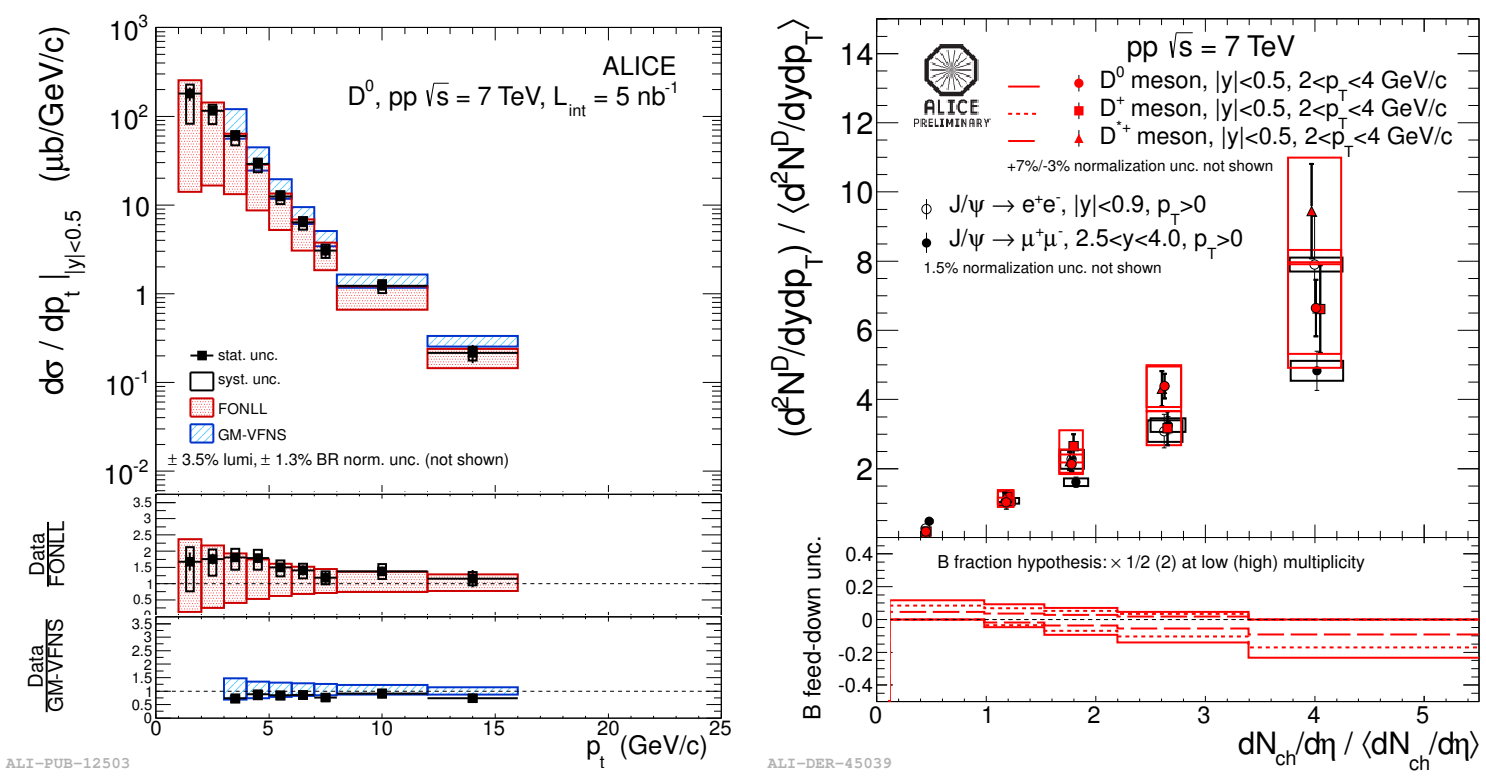

Figure 1. Left: differential cross section of $D^{0}$ mesons compared to FONLL [2-4] and GM-VFNS [5] pQCD calculations [6]. Right: yield of $D^{0}, D^{+}, D^{*+}$ for $2 \mathrm{GeV} / c<p_{\mathrm{T}}<4 \mathrm{GeV} / c$ and $J / \psi$ for $p_{\mathrm{T}}>0$ as function of the event charged particle multiplicity.

total charm cross section has been derived. The measured cross section is in good agreement with next-to-leading order (NLO) pQCD calculations. In addition to the mesonic channels, ALICE also investigates the charmed baryon $\Lambda_{c}$ in the channels $\Lambda_{c} \rightarrow p K^{-} \pi^{+}$and $\Lambda_{c} \rightarrow p K_{s}^{0}$. A signal is seen in both channels mentioned here (Fig. 2).

The production of charmed mesons is also studied as a function of the charged particle multiplicity for different charmed mesons in several $p_{\mathrm{T}}$ intervals. Fig. 1 shows the yield of $D^{0}, D^{+}$, and $D^{*+}$ for $2 \mathrm{GeV} / c<p_{\mathrm{T}}<4 \mathrm{GeV} / c$ as a function of the event charged particle multiplicity. In addition, the dependence of the yield of inclusive $J / \psi$, containing contributions from both prompt and non-prompt $J / \psi$, for $p_{\mathrm{T}}>0$ on the event charged particle multiplicity is shown in the same figure. A similar increase in the yield with multiplicity is seen for all the channels. This

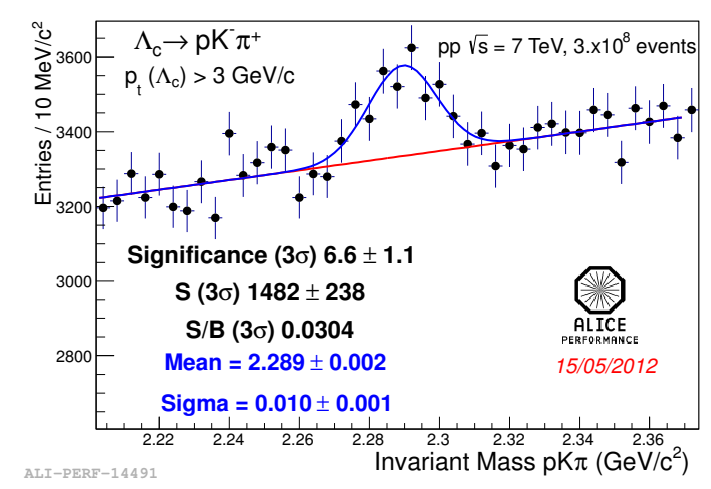

Figure 2. Invariant mass of the $\Lambda_{c}$ in the channel $\Lambda_{c} \rightarrow p K^{-} \pi^{+}$ for $p_{\mathrm{T}}>3 \mathrm{GeV} / \mathrm{c}$. indicates that multi-parton interactions also affect the hard momentum scales relevant for charm production.

\section{Measurement in the semi-leptonic channels}

In addition to the measurements in the hadronic channels, ALICE also measures heavy-flavour production via the semi-electronic channel at midrapidity. Since this is an inclusive measurement, the electron sample contains background from various sources, dominantly $\gamma$ conversions and $\pi^{0}$ Dalitz-decays, which is estimated using a cocktail approach and subtracted [9]. As shown in Fig 3, the differential cross section is well described by FONLL pQCD calculations.

Several methods are available to extract the differential cross section of electrons from beauty hadron decays:

- Selection of tracks with a large radial distance to the primary vertex due to the larger lifetime of beauty hadrons [11]

- Usage of azimuthal angular correlations between electrons and hadrons

\section{- Reconstruction of secondary vertices}

The differential cross section of electrons from beauty hadron decays is shown in comparison to FONLL pQCD calculations in Fig. 3 (right). The cross section is obtained using the first method at up to $p_{\mathrm{T}}=8 \mathrm{GeV} / c$ and the third method for $p_{\mathrm{T}}>8 \mathrm{GeV} / c$. The result is well described by the calculation. The total cross section, obtained by extrapolating the measured cross section to the full phasespace, is found to be in good agreement with next-toleading order pQCD calculations[11]. 

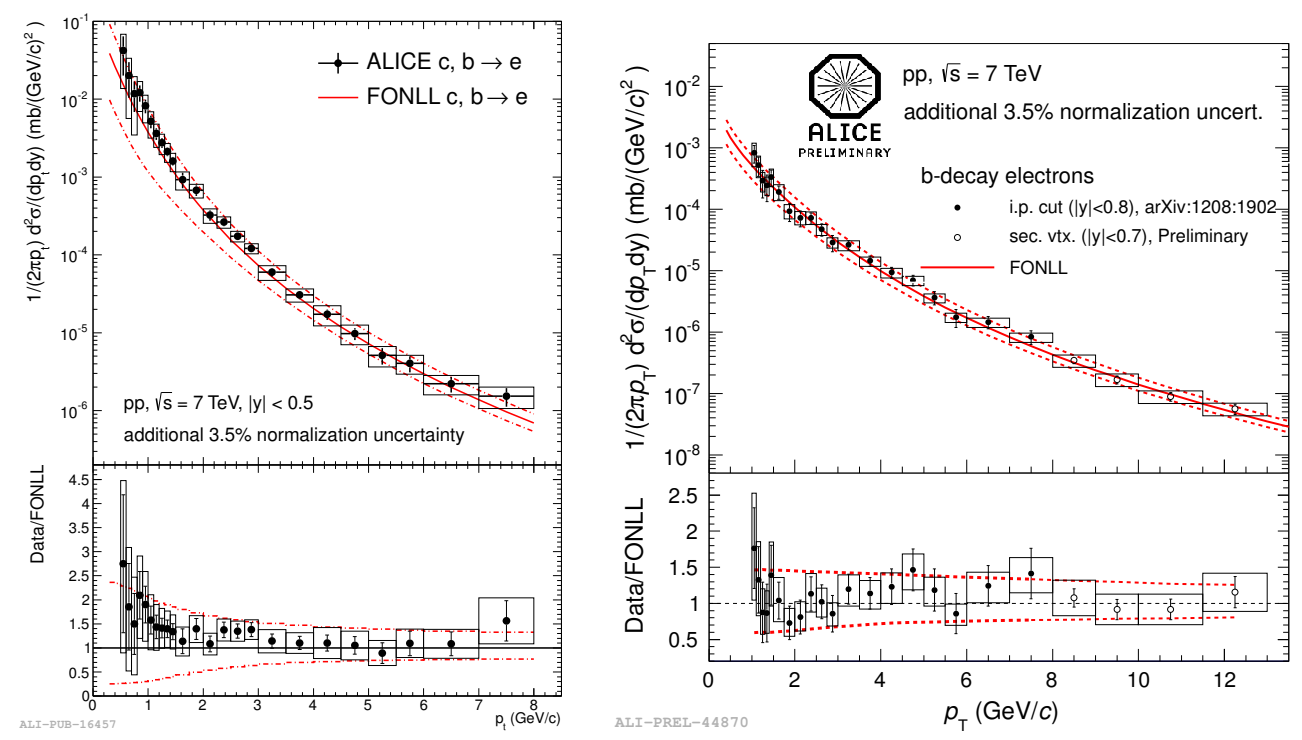

Figure 3. Left: differential cross section of electrons from semi-leptonic heavy-flavour hadron decays compared to FONLL pQCD calculations [9]. Right: differential cross section of electrons from beauty hadron decays compared to a FONLL pQCD calculation [11].
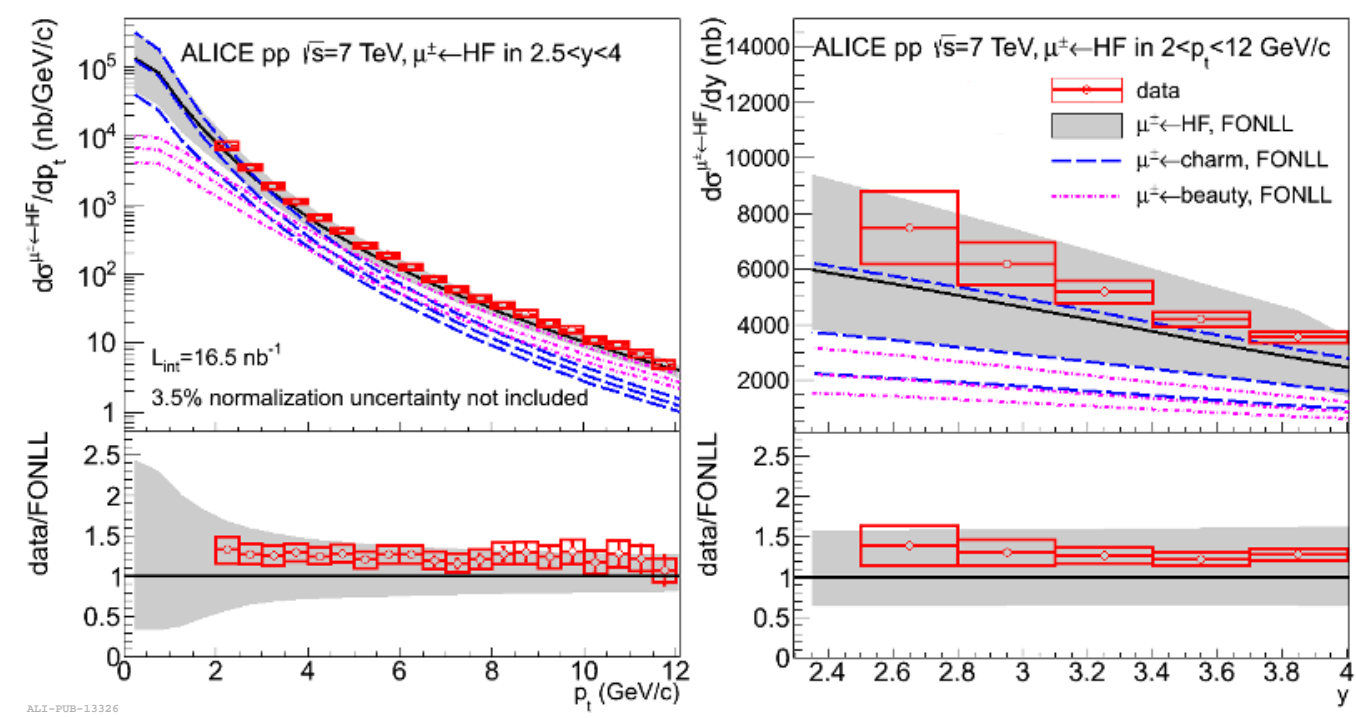

Figure 4. Left: $p_{\mathrm{T}}$-differential cross section of muons from heavy-flavour hadron decays in $2.5<\mathrm{y}<4$. Right: y-differential cross section of muons from heavy-flavour hadron decaysat forward rapidity [10]

At forward rapidity, heavy-flavour production is measured in the semi-muonic channel. Also the muon sample contains background from various sources (i.e. hadron decays), which is estimated normalising the $p_{\mathrm{T}}$-shape obtained from Monte-Carlo simulations to the measured yield at low $p_{\mathrm{T}}$ and subtracted. The $p_{\mathrm{T}^{-}}$and y-differential cross section is well-described by FONLL pQCD calculations. (Fig. 4) [10].

\section{Measurements of heavy-flavour production in pp-collisions at $\sqrt{s}=2.76 \mathrm{TeV}$}

Heavy-flavour measurements have also been performed at $\sqrt{s}=2.76 \mathrm{TeV}$, the reference energy for $\mathrm{Pb}-\mathrm{Pb}$ collisions at the LHC in 2010 and 2011. The differential cross section has been measured for different $\mathrm{D}$ meson channels (Fig. 5 (left)) [12], electrons from semi- leptonic heavyflavour hadron decays at midrapidity (Fig. 5 (middle)) and muons from semi-leptonic heavy-flavour hadron decays at forward rapidity (Fig. 5 (right)) [14]. As observed in pp collisions at $\sqrt{s}=7 \mathrm{TeV}$, FONLL pQCD calculations are 

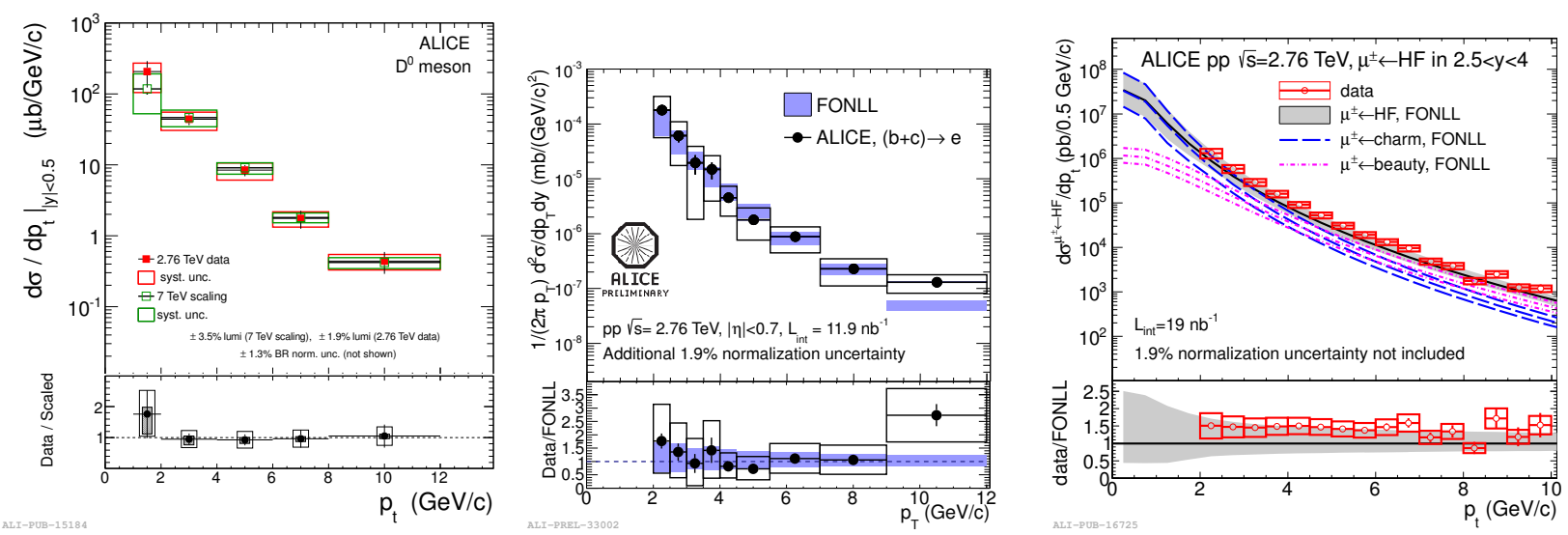

Figure 5. Measurement of heavy-flavour production in pp-collisions at $\sqrt{s}=2.76 \mathrm{TeV}$. Left: differential cross section of $D^{0}$ mesons [12] compared to the cross section at $\sqrt{s}=7 \mathrm{TeV}$ scaled to $\sqrt{s}=2.76 \mathrm{TeV}$ using a pQCD-driven approach [13]. Middle: differential cross section of electrons from semi-leptonic heavy-flavour hadron decays at midrapidity compared to a FONLL pQCD calculation. Right: differential cross section of muons from semi-leptonic heavy-flavour hadron decays at forward rapidity compared to a FONLL pQCD calculation [14].

in good agreement with the measurement. This allows to perform a $\mathrm{pQCD}$-driven scaling procedure to obtain reference spectra for different channels based on the spectra measured at the higher energy [13], either to improve the precision of the reference or to provide a reference in a $p_{\mathrm{T}}$ region where the statistics of the dataset at $2.76 \mathrm{TeV}$ is not sufficient to provide a measurement.

Reference spectra are important in order to investigate the behaviour of the hot and dense medium created in heavy-ion collisions [15]. For this, $\mathrm{Pb}-\mathrm{Pb}$ collisions are compared to $\mathrm{pp}$ collisions using the nuclear modification factor $R_{A A}$ defined as

$$
R_{A A}=\frac{1}{<T_{A A}>} \frac{d N^{P b P b} / d p_{\mathrm{T}}}{d \sigma^{P p} / d p_{\mathrm{T}}}
$$

where $d N^{P b P b} / d p_{\mathrm{T}}$ is the yield in $\mathrm{Pb}-\mathrm{Pb}$ collisions, $d \sigma^{p p} / d p_{\mathrm{T}}$ is the cross section in pp collisions, and $<$ $T_{A A}>$ is the nuclear overlap function. In case there is no effect of the medium, the nuclear modification factor is 1 .

The nuclear modification factor $D^{0}, D^{+}, D^{*}[16]$ and $D_{s}^{+}$in central $\mathrm{Pb}-\mathrm{Pb}$ collisions at $\sqrt{s_{N N}}=2.76 \mathrm{TeV}$ is shown in Fig. 6. A suppression is seen at high $p_{\mathrm{T}}$ [17]. The nuclear modification factor is used to study partonic energy loss in the hot and dense medium.

\section{Conclusions}

The production of heavy flavours was measured in pp collisions at $\sqrt{s}=7 \mathrm{TeV}$ and $\sqrt{s}=2.76 \mathrm{TeV}$ in hadronic channels as well as in semi-leptonic channels by ALICE. The various measurements show a good agreement with pQCD calculations. The measurements serve as a baseline to study properties of the hot and dense medium created in $\mathrm{Pb}-\mathrm{Pb}$ collisions. Furthermore the charm production as a function of the event charged particle multiplicity, which is sensitive to multiple hard parton collisions, is studied in pp

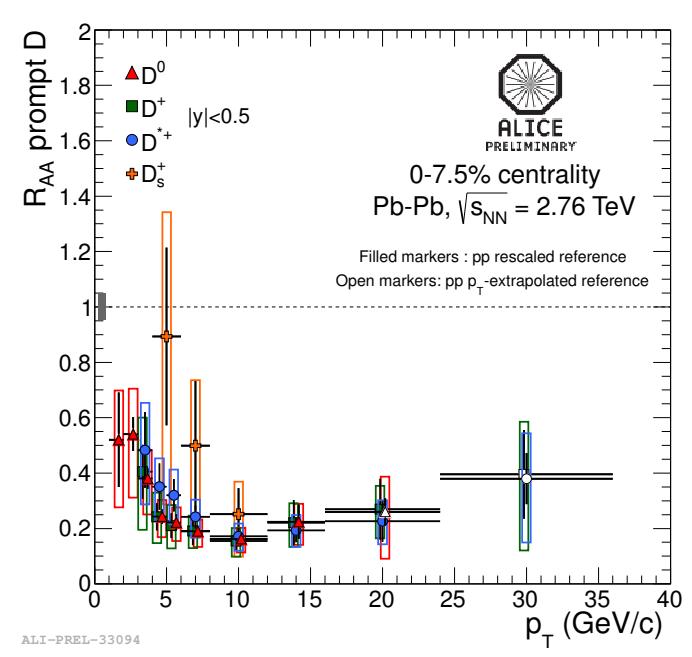

Figure 6. Nuclear modification factor of $D^{0}, D^{+}, D^{*}[16]$ and $D_{s}^{+}$ in central $\mathrm{Pb}-\mathrm{Pb}$ collisions at $\sqrt{s_{N N}}=2.76 \mathrm{TeV}$.

collisions at $\sqrt{s}=7 \mathrm{TeV}$. Here an increase with the multiplicity is observed for different charmed meson species in several $p_{\mathrm{T}}$ bins.

\section{References}

[1] K. Aamodt et al. (ALICE Collaboration), JINST 3, S08002 (2008)

[2] M. Cacciari, M. Greco, P. Nason, JHEP 9805, 007 (1998), hep-ph/9803400

[3] M. Cacciari, S. Frixione, P. Nason, JHEP 0103, 006 (2001), hep-ph/0102134

[4] M. Cacciari, S. Frixione, N. Houdeau, M.L. Mangano, P. Nason et al., JHEP 1210, 137 (2012), 1205.6344

[5] B. Kniehl, G. Kramer, I. Schienbein, H. Spiesberger, Eur.Phys.J. C72, 2082 (2012), 1202 . 0439 
[6] B. Abelev et al. (ALICE Collaboration), JHEP 1201, 128 (2012), 1111.1553

[7] B. Abelev et al. (ALICE Collaboration), Phys.Lett. B718, 279 (2012), 1208. 1948

[8] R. Maciula, A. Szczurek, Phys.Rev. D87, 094022 (2013), 1301.3033

[9] B. Abelev et al. (ALICE Collaboration), Phys.Rev. D86, 112007 (2012), 1205.5423

[10] B. Abelev et al. (ALICE Collaboration), Phys.Lett. B708, 265 (2012), 1201. 3791

[11] B. Abelev et al. (ALICE Collaboration), Phys.Lett. B721, 13 (2013), 1208. 1902
[12] B. Abelev et al. (ALICE Collaboration), JHEP 1207, 191 (2012), 1205.4007

[13] R. Averbeck, N. Bastid, Z.C. del Valle, P. Crochet, A. Dainese et al. (2011), 1107. 3243

[14] B. Abelev et al. (ALICE Collaboration), Phys.Rev.Lett. 109, 112301 (2012), 1205.6443

[15] A. Rossi (ALICE Collaboration), these proceedings

[16] B. Abelev et al. (ALICE Collaboration), JHEP 1209, 112 (2012), 1203.2160

[17] C. Bianchin (ALICE Collaboration), these proceedings 